\title{
Influência das temperaturas de secagem nas características físico-químicas da polpa do baru
}

\section{Influence of drying temperatures on physicochemical characteristics of baru pulp}

\author{
Flávia Alves Morais \\ Instituto Federal Goiano - Campus Rio Verde \\ E-mail: fa.a.mrs@gmail.com \\ OrclD: https://orcid.org/0000-0003-2741-1590
}

Celso Martins Belisário

Instituto Federal Goiano - Campus Rio Verde

E-mail: celso.belisario@ifgoiano.edu.br

OrcID: https://orcid.org/0000-0001-8693-6384

Rogério Favareto

Instituto Federal Goiano - Campus Rio Verde

E-mail: rogerio.favareto@ifgoiano.edu.br

OrclD: https://orcid.org/0000-0001-5293-0451

Geisa Priscilla Araújo Gomes Maia Instituto Federal Goiano - Campus Rio Verde

E-mail: geisamaia@hotmail.com

OrclD: https://orcid.org/0000-0001-9273-2185

Vicente Douglas Figueredo Carvalho Instituto Federal Goiano - Campus Rio Verde

E-mail: vicente99vd@gmail.com

OrclD: https://orcid.org/0000-0002-2652-0552

Resumo: O barueiro (Dipteryx alata Vogel) é uma espécie frutífera do Cerrado, que produz o baru, uma drupa de coloração marrom, formato ovoide e abriga no seu interior uma única amêndoa. Sendo a amêndoa o principal produto, a polpa e o endocarpo lenhoso são descartados. Considerando as potencialidades desse material e seguindo a tendência da minimização do desperdício de alimentos, quantificaram-se os compostos fenólicos totais, flavonoides, taninos, $\mathrm{pH}$, acidez total titulável, sólidos solúveis totais e atividade antioxidante da polpa do baru submetida à secagem a 50,60,70 e $80^{\circ} \mathrm{C}$. A amostra seca a $80^{\circ} \mathrm{C}$ apresentou o menor teor de flavonoides. Taninos e fenólicos totais não se diferiram entre as temperaturas de secagem, e a atividade antioxidante apresentou menor proteção na polpa seca a $50{ }^{\circ} \mathrm{C}$. A secagem da polpa do baru em temperaturas entre 60 e $70^{\circ} \mathrm{C}$ é viável para a preservação das potencialidades bioativas e físico-químicas.

Palavras-chave: Dipteryx alata Vogel. Fenólicos totais. Flavonoides. Pós-colheita. Taninos.

Abstract: The barueiro (Dipteryx alata Vogel) is a fruiting Cerrado species, which produces the baru, an ovoid form brown drupe that contains a single almond inside. Since almond is the main product, the pulp and woody endocarp are discarded. Considering the potential of this material, and following the trend of minimizing food waste, Were quantified the total phenolic compounds, flavonoids, tannins, $\mathrm{pH}$, total titratable acidity, total soluble solids and antioxidant activity of baru pulp submitted to drying at $50,60,70$ and $80^{\circ} \mathrm{C}$. The dry sample at $80{ }^{\circ} \mathrm{C}$ presented the lowest flavonoid content. Tannins and total phenolics did not differ between drying temperatures, and antioxidant activity showed lower protection at pulp dried at $50{ }^{\circ} \mathrm{C}$. The drying of baru pulp in temperatures between 60 and $70{ }^{\circ} \mathrm{C}$ is viable for preserving of bioactive potential and physicochemical.

Keywords: Dipteryx alata Vogel. Total phenolics. Flavonoids. Postharvest. Tannins. 


\section{Introdução}

Nos últimos anos, a sociedade tem acompanhado uma crescente busca por alimentos fornecedores de energia e nutrientes que além da qualidade sensorial também promovam benefícios para a saúde. Dentre esses nutrientes, destacam-se os compostos com atividade antioxidante presentes em grande quantidade em frutos do Cerrado (Rocha, Figueiredo, Araújo \& Moreira-Araújo, 2013; Alves, Dias, Hassimotto \& Naves, 2017).

Dentre as frutíferas nativas do Cerrado brasileiro destaca-se o baru, fruto do barueiro (Dipteryx alata Vogel), com formato ovoide revestido de polpa e internamente constituído de uma amêndoa com ampla utilização na fabricação de doces (Siqueira et al., 2013). Contudo, a parte consumida e valorizada deste fruto é a amêndoa, e dessa forma, grandes quantidades de resíduos são geradas no descarte da polpa juntamente com o endocarpo lenhoso. Através do aproveitamento integral do fruto, é possível aumentar as possibilidades de ingestão de diversos nutrientes e ainda contribuir com a redução da geração de resíduos (Lima, Tavares, Costa \& Pierucci, 2014).

A casca e a polpa do baru, que são comumente descartadas, apresentam compostos bioativos como, por exemplo, os compostos fenólicos que possuem potencial antioxidante, fibras, macronutrientes e micronutrientes (Marcelino, Coleta, Candido \& Santos, 2018). Nessas classes de compostos os flavonoides que segundo Silva et al. (2014), são encontrados em maiores proporções na polpa das frutas e vegetais, se destacam por atuarem na interrupção das reações dos radicais livres, por meio de ligações de hidrogênio ou interações com pares de elétrons livres (Silva et al., 2016).

Além dos atributos nutricionais, há relatos do uso da polpa do baru para fins terapêuticos. Em um desses trabalhos, reporta-se que quando macerada ou aquecida, a polpa produz efeitos terapêuticos que reduzem dores musculares, previnem a osteoporose e o reumatismo (Ribeiro, Bieski, Balogun \& Martins, 2017).

No contexto da importância dos compostos bioativos, das práticas de redução do desperdício de alimentos e no incentivo ao consumo de frutos nativos, o objetivo deste trabalho foi verificar a influência das temperaturas de secagem da polpa do baru no teor de fenólicos totais, flavonoides, taninos, atividade antioxidante, $\mathrm{pH}$, sólidos solúveis totais e acidez total titulável.

\section{Material e Métodos}

Os frutos foram coletados de caída, em barueiros localizados no município de Acreúna-GO, a $17^{\circ} 23^{\prime} 44^{\prime \prime} \mathrm{S}$ e $50^{\circ} 22^{\prime} 37^{\prime \prime} \mathrm{O}$ e altitude de 542 metros. Foram selecionados de acordo com a integridade do pericarpo (epicarpo, mesocarpo e endocarpo) e boas práticas de manejo elaboradas pelo do Ministério da Agricultura, Pecuária e Abastecimento (MAPA, 2012) e encaminhados para o Laboratório de Fitoquímica do IFGoiano - Campus Rio Verde. Em seguida foram sanitizados em solução de hipoclorito de sódio a $10 \%$, e a polpa (epicarpo e mesocarpo) foi retirada manualmente com facas de aço previamente sanitizadas, acondicionada em embalagens de polipropileno, fechadas a vácuo e armazenados em freezer convencional sob temperatura aproximada de $-18^{\circ} \mathrm{C}$ até o início dos experimentos.

O teor de água inicial do baru foi determinado pela perda de massa de amostras frescas da polpa, por aquecimento em estufa com circulação de ar a $105^{\circ} \mathrm{C}$ até massa constante (Instituto Adolf Lutz [IAL], 2006). Para a secagem, foram feitas camadas de $5 \mathrm{~cm}$ da polpa de baru, dispostas em bandejas de inox e inseridas em estufa com circulação de ar forçada nas temperaturas de $50,60,70$ e $80{ }^{\circ} \mathrm{C}$, até massa constante. As amostras secas foram retiradas aleatoriamente e submetidas às análises de $\mathrm{pH}$, com pHmetro, sólidos solúveis totais em ${ }^{\circ}$ Brix por refratômetro digital de bancada, acidez total titulável (\%) por titulação com $\mathrm{NaOH}$ $0,1 \mathrm{~mol} \mathrm{~L}^{-1}$, de acordo com as metodologias propostas pelo Instituto Adolfo Lutz (IAL, 2008).

As extrações para a determinação de taninos, compostos fenólicos totais e flavonoides, foram feitas de acordo com Barbosa et al. (2001). Em cerca de $3 \mathrm{~g}$ de polpa seca e triturada foram adicionados $100 \mathrm{~mL}$ de solução hidroetanólica $50 \%$ (v/v) e mantidas em repouso, ao abrigo da luz, por 24 horas. Em seguida foram filtradas, transferidas para balões de $100 \mathrm{~mL}$ e o volume completado com a solução extratora. 
Os flavonoides totais foram quantificados pelo método colorimétrico descrito por Jia, Tang e Wu (1999). Em tubos de ensaio, alíquotas de 0,5 mL dos extratos foram adicionados de 0,3 $\mathrm{mL}$ de $\mathrm{NaNO}_{2}$ (aq) a $5 \%, 0,3 \mathrm{~mL}$ de $\mathrm{AlCl}_{3}$ (metanólico) a10\% e $2 \mathrm{~mL}$ de $\mathrm{NaOH}(\mathrm{aq}) 1 \mathrm{~mol} \mathrm{~L}^{-1}$. Após agitação, cada tubo ficou em repouso por 10 minutos e efetuou-se a leitura em espectrofotômetro Uv-Vis a $510 \mathrm{~nm}$, previamente zerado com água destilada. As concentrações foram calculadas a partir da equação gerada por uma curva padrão de pirocatequina (10 a $50 \mathrm{mg} \mathrm{L}^{-1}$ ), e expressas em mg de pirocatequina $100 \mathrm{~g}^{-1}$.

As concentrações de taninos e de compostos fenólicos totais foram determinadas de acordo com metodologia descrita por Makkar (2000). Em tubos de ensaio foram adicionados 8,2 $\mathrm{mL}$ de água, 0,3 $\mathrm{mL}$ de extrato, $0,5 \mathrm{~mL}$ do reagente Folin - Denis e $1 \mathrm{~mL}$ de solução aquosa de carbonato de sódio 7,5\% (m/v). Após agitação, e subsequente repouso por 30 minutos, foram realizadas leituras de absorbância em espectrofotômetro a $760 \mathrm{~nm}$. Os compostos fenólicos totais foram calculados a partir da função gerada pela curva padrão de ácido tânico (20 a $100 \mathrm{mg} \mathrm{L}^{-1}$ ) e expressos em mg EAT (Equivalente de Ácido Tânico) $100 \mathrm{~g}$

1. O teor de taninos totais foi expresso em percentual relativo ao extrato.

A atividade antioxidante total foi determinada de acordo com a metodologia de Mensor et al. (2001), utilizando-se como soluções extratoras, álcool metílico $50 \%$ e acetona $70 \%$. Comparou-se a capacidade do extrato em reduzir o radical DPPH (2,2-difenil-1-picrilhidrazila) $0,30 \mathrm{mmol} \mathrm{L}^{-1}$, com a atividade do antioxidante sintético Trolox (6-Hidroxi-2,5,7,8-tetrametilchroman-2-ácido carboxílico) a 100 ppm, usado como controle positivo.

As amostras foram preparadas adicionando-se $2,5 \mathrm{~mL}$ do extrato a $1 \mathrm{~mL}$ de solução $\mathrm{DPPH}$, e os brancos das amostras foram constituídos por $2,5 \mathrm{~mL}$ do extrato e $1 \mathrm{~mL}$ da solução extratora. Para o controle positivo, misturou-se 2,5 mL de Trolox e $1 \mathrm{~mL}$ de $\mathrm{DPPH}$, e seu branco constituído por 2,5 $\mathrm{mL}$ de Trolox e 1 $\mathrm{mL}$ de etanol. O controle negativo das amostras e do controle positivo, foi preparado com $2,5 \mathrm{~mL}$ da solução de etanol e $1 \mathrm{~mL}$ de DPPH. Após o preparo, as soluções foram armazenadas por 30 minutos em local escuro. Posteriormente, a determinação das absorbâncias foi realizada em espectrofotômetro a 518 ฤm. A atividade antioxidante foi calculada a partir da Eq. 1.

$$
A A(\%)=100-\left[\frac{(\text { Abs (amostra) }- \text { Abs }(\text { branco }))}{\text { Abs(controle negativo) }}\right] * 100
$$

Onde: Abs(amostra) - absorbância da amostra; Abs(branco) - absorbância do branco; Abs(controle negativo) - absorbância do controle negativo.

Para o cálculo da atividade antioxidante do Trolox, usado como controle positivo, simplesmente substitui-se a Abs (amostra) pela absorbância do controle positivo.

O delineamento foi inteiramente casualizado, com quatro tratamentos constituídos pelas temperaturas de secagem $\left(50,60,70\right.$ e $\left.80^{\circ} \mathrm{C}\right)$. Os experimentos foram realizados em triplicata, submetidos a análise de variância e as médias foram submetidas ao teste de Tukey a $5 \%$ de significância. $O$ software utilizado para os resultados foi o SISVAR, versão 5.6.

\section{Resultados e Discussão}

$\mathrm{Na}$ Tabela 1 estão apresentados os resultados das análises físico-químicas das polpas do baru submetidas à secagem em diferentes temperaturas. Os frutos apresentaram teor de água inicial de 15,2 \pm $1,5 \%$. Após a secagem, as polpas apresentaram teores de água próximos a $10 \%$, indicando que as temperaturas utilizadas não influenciaram na umidade de equilíbrio, já que todas as amostras foram retiradas após apresentarem massas constantes.

O valor médio de umidade do produto de caída foi próximo ao calculado por Alves, Mendonça, Caliari e Cardoso-Santiago (2010) de 13,76\%. Os produtos secos a 50 e $60{ }^{\circ} \mathrm{C}$ tiveram tempo de secagem de $9 \mathrm{~h}$, e a 70 e $80^{\circ} \mathrm{C}$ precisaram de $7 \mathrm{~h}$. Essa diferença era esperada, já que temperaturas mais altas promovem a retirada de água mais rapidamente.

Os teores de sólidos solúveis totais não apresentaram diferença. As condições de secagem não interferiram na concentração desses compostos, tendência observada também por Duarte et al. (2017) em secagem com outras temperaturas.

Em estudo desenvolvido por Fraguas et al. (2014), o conteúdo de sólidos solúveis em amêndoas de baru liofilizadas $\left(3,26^{\circ} \mathrm{BRIX}\right)$ apresentou-se maior quando comparado com as amêndoas torradas $(2,47$ 
${ }^{\circ} \mathrm{BRIX)}$, estes valores são inferiores ao do presente estudo, pois a polpa contém propriedades diferentes das amêndoas.

Tabela 1. Médias e desvios padrão de sólidos solúveis totais (SST), acidez total titulável (ATT) e pH das polpas do baru submetidas a diferentes temperaturas de secagem.

\begin{tabular}{cccc}
\hline Temperaturas de secagem $\left({ }^{\circ} \mathrm{C}\right)$ & SST $\left({ }^{\circ} \mathrm{BRIX}\right)$ & ATT $(\%)$ & $\mathrm{pH}$ \\
\hline 50 & $3,36 \pm 0,42 \mathrm{a}$ & $0,80 \pm 0,01 \mathrm{~b}$ & $8,2 \pm 0,09 \mathrm{a}$ \\
60 & $3,23 \pm 0,21 \mathrm{a}$ & $0,72 \pm 0,05 \mathrm{~b}$ & $8,6 \pm 0,11 \mathrm{a}$ \\
70 & $3,80 \pm 0,6 \mathrm{a}$ & $0,92 \pm 0,10 \mathrm{a}$ & $6,4 \pm 0,10 \mathrm{~b}$ \\
80 & $3,90 \pm 0,82 \mathrm{a}$ & $0,95 \pm 0,04 \mathrm{a}$ & $6,2 \pm 0,09 \mathrm{~b}$ \\
$\mathrm{CV}(\%)$ & 15,62 & 6,67 & 1,24 \\
\hline
\end{tabular}

Médias seguidas das mesmas letras, na mesma coluna, não diferem entre si ao nível de $5 \%$ de significância pelo teste de Tukey.

Ribeiro, Ascheri e Ascheri (2011) realizaram a secagem do mesocarpo do baru a $40{ }^{\circ} \mathrm{C}$, verificaram que o aquecimento proporcionou a potencialização do conteúdo de sólidos solúveis e produziu uma bebida fermentada através da adição do mesocarpo do baru seco e o teor de sólidos solúveis após o preparo da bebida correspondeu a $10,7^{\circ} \mathrm{BRIX}$. Este valor é superior ao do presente estudo, provavelmente porque foi medido após a fermentação disponibilizando mais componentes solúveis detectados no refratômetro.

A acidez total titulável aumentou com o aumento da temperatura de secagem e, consequentemente, acarretou menores valor de $\mathrm{pH}$. O parâmetro de acidez é importante para a conservação dos alimentos, pois reduz os riscos de contaminação microbiológica (Rigueto et al., 2019). O presente trabalho está em concordância com o estudo desenvolvido por Oliveira, Resende e Silva (2018) em que os frutos de baru se tornaram mais ácidos quando se aumentou a temperatura de secagem.

De acordo com Costa, Pinto e Soares (2017), o processo de secagem acarreta a eliminação das moléculas de água e dessa maneira promove a redução dos valores de $\mathrm{pH}$ através do aumento da concentração de ácidos orgânicos nas amostras. Este fato pode ser justificado devido à reação de Maillard com açúcares em altas temperaturas (Muzaffar \& Kumar, 2015). Dessa forma, as polpas secas sob temperaturas de 70 e $80^{\circ} \mathrm{C}$, respeitando outras condições de armazenamento, podem ter menos riscos de sofrerem perdas causadas por micro-organismos e terem maior vida útil.

$\mathrm{Na}$ Tabela 2 estão apresentados os resultados das análises de flavonoides, taninos, fenólicos totais e atividade antioxidante da polpa do baru. As concentrações de flavonoides encontradas na polpa do baru foram maiores do que as verificadas por Siqueira et al. (2013) e próximos aos observados por Rocha et al. (2013) em extratos hidroetanólicos de cajuzinho do Cerrado.

Tabela 2. Concentrações de flavonoides, taninos, fenólicos totais e atividade antioxidante da polpa do baru proveniente de diferentes temperaturas de secagem.

\begin{tabular}{ccccc}
\hline $\begin{array}{c}\text { Temperatura } \\
\left({ }^{\circ} \mathrm{C}\right)\end{array}$ & Flavonoides $\left({\left.\mathrm{mg} 100 \mathrm{~g}^{-1}\right)}^{\text {Taninos }(\%)}\right.$ & $\begin{array}{c}\text { Fenólicos Totais } \\
(\text { mg EAT 100 g }\end{array}$ & $\begin{array}{c}\text { Atividade Antioxidante } \\
(\%)\end{array}$ \\
\hline 50 & $2,81 \pm 0,80 \mathrm{a}$ & $14,03 \pm 0,40 \mathrm{a}$ & $42,11 \pm 1,20 \mathrm{a}$ & $64,03 \pm 7,00 \mathrm{~b}$ \\
60 & $2,53 \pm 0,24 \mathrm{a}$ & $14,81 \pm 0,50 \mathrm{a}$ & $44,45 \pm 1,60 \mathrm{a}$ & $91,97 \pm 1,00 \mathrm{a}$ \\
70 & $2,97 \pm 1,1 \mathrm{a}$ & $13,90 \pm 0,37 \mathrm{a}$ & $41,70 \pm 1,12 \mathrm{a}$ & $93,41 \pm 2,70 \mathrm{a}$ \\
80 & $1,59 \pm 0,48 \mathrm{~b}$ & $14,82 \pm 0,82 \mathrm{a}$ & $44,47 \pm 2,50 \mathrm{a}$ & $88,13 \pm 0,30 \mathrm{a}$ \\
CV $(\%)$ & 16,44 & 3,88 & 3,88 & 4,45 \\
\hline
\end{tabular}

Médias seguidas das mesmas letras, na mesma coluna, não diferem entre si ao nível de $5 \%$ pelo teste de Tukey.

As variações das concentrações de flavonoides de acordo com as temperaturas de secagem, seguem as observadas por Souza, Fernandes, Bott e Oliveira (2015) em trabalho com extratos vegetais secos sob diferentes temperaturas. Em estudo de Fraguas et al. (2014), verificaram que quando liofilizadas, as amêndoas apresentaram teores de flavonoides de $9,3 \mathrm{mg} 100 \mathrm{~g}^{-1}$ e torradas a $150{ }^{\circ} \mathrm{C}, 1,61 \mathrm{mg}^{\circ} 100 \mathrm{~g}^{-1}$. A mesma tendência pôde ser verificada neste trabalho, pois, a $80{ }^{\circ} \mathrm{C}$ houve redução dos flavonoides. Este decréscimo é devido à facilidade de degradação desses compostos em temperaturas elevadas, indicando que o método de secagem deve considerar temperaturas de até $70{ }^{\circ} \mathrm{C}$ para manter os melhores níveis de flavonoides, que são constituintes considerados bioativos e com capacidade de sequestrar radicais livres (Santiago et al., 2018). 
As maiores atividades antioxidantes foram verificadas nas amostras secas a 60,70 e $80{ }^{\circ} \mathrm{C}$. Pelo fato de os flavonoides serem um dos grupos responsáveis pela atividade antioxidante, mas serem de fácil degradação, tanto as temperaturas mais elevadas, quanto maiores tempos de exposição em baixas temperaturas podem acarretar na diminuição dessa propriedade (Azeez et al., 2019).

Embora os taninos sejam considerados antinutricionais, pesquisas tem demonstrado que são boas fontes de compostos antioxidantes. De acordo com Siqueira, Castro, Silveira e Lourenço (2016), os teores de taninos decrescem com a maturação e estão associados a adstringência dos frutos e podem ter suas concentrações reduzidas através dos processos de aquecimento, como por exemplo a secagem, mas no intervalo de temperatura utilizado neste estudo, não houve decréscimo.

O teor de fenólicos totais não apresentou diferença significativa nas temperaturas de secagem utilizadas, sendo menores que os encontrados por Siqueira et al. (2013). Estas variações podem ser devido à origem geográfica dos frutos, estádio de maturação, atributos do solo, dentre outros fatores.

As variações de temperaturas de secagem entre 50 e $80^{\circ} \mathrm{C}$ não foram suficientes para degradar os compostos fenólicos. Santiago et al. (2018) analisaram a influência do tratamento térmico na quantidade de compostos fenólicos e capacidade antioxidante do baru presentes na polpa e amêndoas cruas e torradas. Os resultados expressaram quantidades superiores de fenólicos e maior capacidade antioxidante na amêndoa crua quando comparada com a amêndoa torrada a $140^{\circ} \mathrm{C}$. Em temperaturas de torrefação, é previsto que esses compostos sofram degradação e apresentem concentrações menores se comparados com as amostras frescas, e como verificado, menores do que em amostras secas entre 50 e $80^{\circ} \mathrm{C}$. .

\section{Conclusão}

Polpas de baru secas a 60 e $70{ }^{\circ} \mathrm{C}$ apresentaram maiores valores de acidez, podendo contribuir com a redução da degradação microbiológica em situações de armazenamento. As temperaturas de secagem não influenciaram na concentração de compostos fenólicos totais nem na concentração de taninos. Para a manutenção de maiores teores de flavonoides a temperatura de secagem não pode ser superior a $70^{\circ} \mathrm{C}$.

As atividades antioxidantes foram maiores nas temperaturas de 60,70 e $80{ }^{\circ} \mathrm{C}$, sendo ligeiramente maior nas amostras secas a $70^{\circ} \mathrm{C}$. Para a manutenção de biocompostos e atividade antioxidante, indica-se a secagem da polpa do baru em temperaturas entre 60 e $70{ }^{\circ} \mathrm{C}$. A polpa do baru, tratada como resíduo, pode fornecer substâncias com propriedades bioativas, elevada atividade antioxidante e passando pelo processo de secagem nas temperaturas de 60 e $70^{\circ} \mathrm{C}$ podem ter maior vida útil.

\section{Agradecimentos}

Ao Instituto Federal de Educação, Ciência e Tecnologia Goiano, Campus Rio Verde; ao Laboratório de Fitoquímica; a Central de Análises Multiusuário; CAPES e Programa de Pós Graduação em Tecnologia de Alimentos.

\section{Referências}

Alves, A.M., Dias, T., Hassimotto, N.M.A., Naves, M.M.V. (2017). Ascorbic acid and phenolic contents, antioxidant capacity and flavonoids composition of Brazilian Savannah native fruits. Food Science and Technology, 37(4), 564-569. https://doi.org/10.1590/1678-457x.26716

Alves, A., Mendonça, A., Caliari, M., Cardoso-Santiago, R. (2010). Avaliação química e física de componentes do Baru (Dipteryx alata Vog.) para estudo da vida de prateleira. Pesquisa Agropecuária Tropical, 40(3), 266273.

Azeez, L., Adebisi, S.A., Oyedeji, A.O., Adetoro, R.O., Tijani, K.O. (2019). Bioactive compounds contents, drying kinetics and mathematical modelling of tomato slices influenced by drying temperatures and time. Journal of the Saudi Society of Agricultural Sciences, 18(2), 120-126. https://doi.org/10.1016/j.jssas.2017.03.002

Barbosa, W.L.R., Quignard, E., Tavares, I.C.C., Pinto, L.N., Oliveira, F.Q., Oliveira, R.M. (2001). Manual para Análise Fitoquímica e Cromatográfica de Extratos Vegetais [Manual]. Belém, PA: UFPA. 
Costa, A.P.F., Pinto, E.G., Soares, D.S.B. (2017). Obtaining of the mesocarp meal of pequi. Revista Agrarian, 10(38), 349-354.

Duarte, E.L., Carlos, L.A., Gonçalves, C.R., Andrade, R.M., Oliveira, K.G. (2017). Influência da liofilização sobre os carotenoides de frutos do Cerrado e comportamento higroscópico dos produtos liofilizados. Perspectiva Online: Biologia e Saúde, 23(7), 22-33. https://doi.org/10.25242/886872320171108

Fraguas, R.M., Simão, A.A., Leal, R.S., Santos, C.M., Rocha, D.A., Tavares, T.S., Marques, T.R., Duarte, M.H., Marcussi, S., Abreu, C.M.P. (2014). Chemical composition of processed baru (Dipteryx alata Vog.) almonds: Lyophilization and roasting. African Journal of Agricultural Research, 9(13), 1061-1069. https://doi.org/10.5897 / AJAR2014.8469

Instituto Adolf Lutz (2008). Métodos físico-químicos para análise de alimentos. Recuperado de https://wp.ufpel.edu.br/nutricaobromatologia/files/2013/07/NormasADOLFOLUTZ.pdf .

Jia, Z., Tang, M., Wu, J. (1999). The determination of flavonoid contents in mulberry and their scavenging effects on superoxide radicals. Food Chemistry, 9(4), 555-559. http://doi.org/10.1016/S0308-8146(98)00102-2

Lima, B. N. B., Lima, F. F., Tavares, M. I. B., Costa, A. M.M., Pierucci, A. P. T. R. (2014). Determination of the centesimal composition and characterization of flours from fruit seeds. Food Chemistry, 151, $293-299$. https://doi.org/10.1016/j.foodchem.2013.11.036

Makkar, H. P. S. (2000). Quantification of Tannins in Tree Foliage. Vienna, Austria: FAO/IAEA.

Marcelino, G., Coleta, I.T., Candido, C.J., Santos, E.F. (2018). Caracterização e análise sensorial de cupcakes elaborados com diferentes concentrações de farinha de polpa e polpa de baru (Dipteryx alata Vog.). Revista Multitemas, 23(54), 265-281.

Mensor, L.L., Menezes, F.S., Leitão, G.G., Reis, A.S., Santos, T.C., Coube, C.S., Leitão, S.G. (2001). Screnning of Brazilian plant extracts for antioxidant activity by the use of DPPH free radical method. Phytotherapy Research, 15(2), 127-130. https://doi.org/10.1002/ptr.687

Ministério da Agricultura, Pecuária e Abastecimento. (2012). Boas práticas de manejo para o extrativismo sustentável orgânico.

Recuperado de https://www.gov.br/agricultura/pt-br/assuntos/sustentabilidade/organicos/arquivospublicacoes-

organicos/boas_praticas_de_manejo_para_o_extrativismo_sustentavel_organico_do_baru.pdf/@@download/f ile/boas_praticas_de_manejo_para_o_extrativismo_susten.pdf

Muzaffar, K., Kumar, P. (2015). Parameter optimization for spray drying of tamarind pulp using response surface methodology. Powder Technology, 279, 179-184. https://doi.org/10.1016/j.powtec.2015.04.010

Oliveira, P.M., Oliveira, D.E.C., Resende, O., Silva, D.V. (2018). Study of the drying of mesocarp of Baru (Dipteryx alata Vogel) fruits. Revista Brasileira de Engenharia Agrícola e Ambiental, 22(12), $872-877$. https://doi.org/10.1590/1807-1929/agriambi.v22n12p872-877

Ribeiro, A.E.C., Ascheri, D.P.R., Ascheri, J.L.R. (2011). Aplicação da metodologia de superfície de resposta para a seleção de uma bebida alcoólica fermentada de polpa de baru. Revista Agrotecnologia, 2(1), 57-72. https://doi.org/10.12971/2179-5959.v02n01a05

Ribeiro, R.V., Bieski, I.G.C., Balogun, S.O., Martins, D.T.O. (2017). Ethnobotanical study of medicinal plants used by Ribeirinhos in the North Araguaia 34 microregion, Mato Grosso, Brazil. Journal of Ethnopharmacology, 205, 69-102. https://doi.org/10.1016/j.jep.2017.04.023

Rigueto, C.V.T., Soares, C.C., Brandão, M.V., Alonso, I.C.R., Geraldi, C.A.Q., Machado, F.P., Loss, R.A. (2019). Avaliação da Temperatura de Secagem na elaboração de farinha do caroço do abacate (Persea americana mil). Avanços e Desafios da Nutrição, 1(3), 93-101. https://doi.org/ https://doi.org/10.22533/AT.ED.40819240511

Rocha, M.S., Figueiredo, R.W., Araújo, M.A.M., Moreira-Araújo, R.S.R. (2013). Caracterização físico-química e atividade antioxidante (in vitro) de frutos do Cerrado Piauiense. Revista Brasileira de Fruticultura, 35(4), 933-941. https://doi.org/10.1590/S0100-29452013000400003 
